\title{
POSTER SYMPOSIUM I
}

\section{Platelets: Prostaglandin Synthesis.}

UNIQUE SIGNIFICANCE OF DIHOMO- $\gamma$-LINOLENIC ACID MECHANISMS FOR THE PREVENTION OF THROMBOSIS. A. L. Willis, Roche Products Ltd., Welwyn Garden City, England.

Common anti-platelet aggregatory agents act like aspirin to block cyclo-oxygenation of arachidonic acid (AA) to the endoperoxide $\mathrm{PGH}_{2}$. Alternatively, they act 1 ike $\mathrm{PGE}_{1}$ to increase platelet cyclic AMP (CAMP). Inhibition of platelet production of thromboxane (TX) $\mathrm{A}_{2}$ may also be desirable. Dihomo- $\gamma-1$ inolenic acid (DHLA) apparently acts through these and other mechanisms. Competition of DHLA with AA for vesicular gland cyclo-oxygenase occurred so that antiaggregatory $\mathrm{PGE}_{1}$ and $\mathrm{PGH}_{1}$ were produced at the expense of $\mathrm{PGH}_{2}$ and $\mathrm{PGE}_{2}$ (which together produce aggregation); $\mathrm{O}_{2}$ uptake was unimpaired. Consistent with such an action in platelets, the antiaggregatory effects of DHLA were prevented by aspirin or other inhibitors of cyclo-oxygenase and were enhanced by Ro 21-1724, a selective inhibitor of cAMP phosphodiesterase. Prior incubation of platelet microsomes with DHLA suppressed conversion of AA to a TXA 2 - 1 ike vasoconstrictor substance and to more stable material behaving 1 ike $\mathrm{TXB}_{2}$ on radio-TLC. In addition, DHLA may compete with dietary AA for incorporation into PG precursor pools. The multiple mode of action reported here supports a concept that DHLA is a natural anti-thrombotic factor of key importance.

LECITHIN ANALOG AS A NEW PLATELET AGgREGATING AGENT. J. Hawiger and H.W. Hooper, Jr. Vanderbilt University and VA Hospital, Nashville, Tennessee, USA.

Lecithin comprises $32 \%$ of the human platelet phospholipids which are the source of arachidonic acid required for biosynthesis of prostaglandin endoperoxides, potent inducers of platelet aggregation. A synthetic analog of lecithin, dimethly-DL-2,3-distearoyloxypropy1-2'-hydroxyethylammonium acetate known as a phospholipase $A$ inhibitor, showed a profound in vitro effect upon human platelets. Blood platelets obtained from normal, healthy, fasting volunteers and suspended in autologous plasma aggregated in response to lecithin analog. Platelet aggregation was dose-dependent within the range of $10^{-5}$ to $10^{-4} \mathrm{M}$ of lecithin analog and accompanied by release of $\left[{ }^{3} \mathrm{H}\right]$ serotonin. In contrast, addition of equimolar or higher amounts of lecithin to human platelets in vitro remained without a measurable effect on their function. Sensitivity of human platelets to the lecithin analog used was increased at least 10-fold by separation of platelets from the bulk of plasma proteins by gel filtration on Sepharose $2 \mathrm{~B}$. Release of [ ${ }^{3} \mathrm{H}$ ] serotonin from gel-filtered platelets induced by the lecithin analog studied was dose-dependent and partly reversible due to reuptake of serotonin. Thus, the lecithin analog used in our experiments is a new platelet activating agent which is a "false phospholipid" and phospholipase A inhibitor acting through a previously unrecognized mechanism triggering membrane-mediated func-
tions of human platelets. 